\title{
Addition of chemoradiotherapy to palliative chemotherapy in de novo metastatic nasopharyngeal carcinoma: a real-world study
}

Shuo-Han Zheng ${ }^{1,2+}$, Yu-Tong Wang ${ }^{3 \dagger}$, Song-Ran Liu ${ }^{1,4}$, Zi-Lu Huang ${ }^{1,2}$, Guan-Nan Wang ${ }^{1,5}$, Jin-Tao Lin ${ }^{3}$, Shi-Rong Ding ${ }^{1,2}$, Chen Chen ${ }^{1,2}$ and Yun-Fei Xia ${ }^{1,2^{*}}$

\begin{abstract}
Background: To determine whether concurrent chemotherapy is necessary during locoregional radiotherapy (RT) after palliative chemotherapy (PCT) in patients with de novo metastatic nasopharyngeal carcinoma (mNPC).

Methods: A total of 746 patients with mNPC from 2000 to 2017 at our hospital were retrospectively reviewed. Among them, 355 patients received PCT followed by RT. Overall survival (OS) and progression-free survival (PFS), including locoregional progression-free survival (LRPFS) and distant progression-free survival (DPFS) were estimated with the Kaplan-Meier method and log-rank test. Cox proportional-hazards models, landmark analyses, propensity score matching, and subgroup analyses were used to address confounding.

Results: Of the patients included in our study, 192 received radiotherapy alone after PCT (PCT+RT), and 163 received concurrent chemoradiotherapy after $\mathrm{PCT}(\mathrm{PCT}+\mathrm{CCRT})$. The prognosis of PCT + CCRT was significantly better than that of PCT + RT (5 year OS, 53.0 vs 36.2\%; $P=0.004$ ). After matching, the 5 year OS rates of the two groups were 55.7 and $39.0 \%$, respectively $(P=0.034)$ and the median DPFS were 29.4 and 18.7 months, respectively $(P=0.052)$. Multivariate Cox regression analysis indicated that PCT + CCRT was an independent favorable prognostic factor $(P=0.009)$. In addition, conducting concurrent chemoradiotherapy after 4-6 cycles of PCT or conducting concurrent chemotherapy with single-agent platinum was associated with significant survival benefit in the matched cohort (5 year OS rate, 60.4 or $57.4 \%$, respectively). The survival difference between groups remained significant when evaluating patients who survived for $\geq 1$ year $(P=0.028)$.
\end{abstract}

Conclusions: The optimal treatment strategy of mNPC is the combination of PCT followed by concurrent chemoradiotherapy. More specifically, concurrent chemoradiotherapy with single-agent platinum after 4-6 cycles of PCT is suggested.

Keywords: Nasopharyngeal carcinoma, Metastasis, Chemotherapy, Radiotherapy

*Correspondence: xiayf@sysucc.org.cn

†Shuo-Han Zheng and Yu-Tong Wang equally contributed to this work

${ }^{1}$ State Key Laboratory of Oncology in South China, Collaborative Innovation Center for Cancer Medicine, Guangdong Key Laboratory of Nasopharyngeal Carcinoma Diagnosis and Therapy, Sun Yat-Sen University Cancer Center, Guangzhou, China

Full list of author information is available at the end of the article

\section{Background}

Nasopharyngeal carcinoma (NPC) is a malignant tumor of the nasopharyngeal epithelium that exhibits an unbalanced geographical distribution [1]. In endemic regions, especially in South China, the worldwide age-standardized incidence rate of NPC is up to 25.39/100 000 personyears [2]. Among them, $4-10 \%$ of patients present with de novo metastatic nasopharyngeal carcinoma (mNPC), and original author(s) and the source, provide a link to the Creative Commons licence, and indicate if changes were made. The images or other third party material in this article are included in the article's Creative Commons licence, unless indicated otherwise in a credit line to the material. If material is not included in the article's Creative Commons licence and your intended use is not permitted by statutory regulation or exceeds the permitted use, you will need to obtain permission directly from the copyright holder. To view a copy of this licence, visit http://creativecommons.org/licenses/by/4.0/. The Creative Commons Public Domain Dedication waiver (http://creativeco mmons.org/publicdomain/zero/1.0/) applies to the data made available in this article, unless otherwise stated in a credit line to the data. 
the 5-year survival rate of mNPC is approximately $20 \%$ $[3,4]$. According to the current National Comprehensive Cancer Network (NCCN) guidelines, platinum-based palliative chemotherapy (PCT) with or without locoregional radiotherapy (RT) is the cornerstone of treatment for patients with $\mathrm{mNPC}$ [5]. Many retrospective studies have asserted that cycles of PCT are not always positively related to survival and 4-6 cycles are recommended [6-8]. Moreover, the addition of RT has been shown to significantly benefit the prognosis of patient, increasing the 5-year median overall survival (OS) time to 21-36 months compared with 10-15 months for PCT alone $[1,9,10]$. The sequence and interval of PCT and RT have been explored, and no difference was found between concurrent chemoradiotherapy (CCRT) and PCT followed by RT, or RT initiation within 10 days or even 120 days after PCT $[9,10]$. Recently, a phase III trial comparing the survival of patients with or without $\mathrm{RT}$ in endemic regions demonstrated that RT added to PCT significantly improved OS in chemotherapy-sensitive patients with mNPC (24-month OS, 76.4\% vs 54.5\%; hazard ratio [HR], 0.42 (0.23-0.77); $\mathrm{P}=0.004)$ [11]. However, the details related to combining chemotherapy and radiotherapy, such as whether concurrent chemotherapy is still necessary after PCT or whether the number of PCT cycles can be decreased when RT is applied, remain unclear. Thus, this study aimed to evaluate survival outcomes and identify a promising strategy for mNPC patients treated with PCT and RT.

\section{Methods}

\section{Study population}

Consecutive patients with de novo metastatic nasopharyngeal carcinoma diagnosed at the Sun Yat-sen University Cancer Center between January 1, 2000, and December 31, 2017, were reviewed retrospectively. The eligibility criteria for this study were as follows: (1) biopsy-proven NPC according to the pathological classification system of the World Health Organization (WHO); (2) pathologically or radiologically confirmed distant metastasis at initial diagnosis; (3) treated with PCT followed by RT with or without concurrent chemotherapy; and (4) the absence of other malignant diseases. Patients were excluded if their clinical data were missing, such as the tumor category, node category or metastatic sites. The study cohort ultimately included 355 patients (Additional file 1: Fig. S1).

The study was approved by the Institutional Review Board of Sun Yat-sen University Cancer Center, and informed consent was waived. The study was performed in accordance with institutional policy to protect patients' private information. The authenticity of this article has been validated by uploading the key raw data onto the Research Data Deposit public platform (www. researchdata.org.cn).

\section{Treatment}

All eligible patients received at least one cycle of the following chemotherapy regimens before RT: cisplatin plus 5-fluorouracil (PF, $80 \mathrm{mg} / \mathrm{m}^{2}$ cisplatin intravenously [IV] on day 1 plus $1000 \mathrm{mg} / \mathrm{m}^{2} /$ day 5 -fluorouracil continuous IV infusion on days 1-4), cisplatin plus docetaxel (TP, $75 \mathrm{mg} / \mathrm{m}^{2}$ cisplatin IV on day 1 plus $75 \mathrm{mg} / \mathrm{m}^{2}$ docetaxel IV on day 1), cisplatin plus docetaxel plus 5-fluorouracil (TPF, $75 \mathrm{mg} / \mathrm{m}^{2}$ cisplatin IV on day 1 plus $75 \mathrm{mg} / \mathrm{m}^{2}$ docetaxel IV on day 1 plus $750 \mathrm{mg} / \mathrm{m}^{2} /$ day 5 -fluorouracil continuous IV infusion on days 1-5), or others. All patients underwent RT with two- or three-dimensional conventional radiotherapy, or intensity-modulated radiotherapy (IMRT) on a conventional schedule (5 daily fractions per week). Prescribed doses complied with our center's guidelines with 66-70 Gy for gross tumor volume (GTVnx), 64-70 Gy for the involved cervical lymph nodes (GTVnd), 60-62 Gy for high-risk clinical target volume (CTV1), and 54-56 Gy for low-risk clinical target volume (CTV2) in 30-33 fractions. More details about chemotherapy and radiotherapy were provided in the Supplement (Additional file 1: Description of the radiotherapy and Table S1). The timing and combination of chemotherapy and radiotherapy were determined by the clinicians, patient's condition and willingness. Twentyone days to the commencement of radiotherapy from the end of the last chemotherapy cycle could be considered [11]. Local therapy for metastatic lesions, such as radiotherapy, surgery, transcatheter hepatic artery chemoembolization or radiofrequency ablation, was performed during treatment. The evaluation of tumor response to therapy was based on head and neck magnetic resonance imaging with contrast, chest radiography/chest computed tomography, abdominal sonography/abdominal computed tomography, bone scan or positron emission tomography/computed tomography, and patients were classified as having a complete response (CR), a partial response $(\mathrm{PR})$, stable disease $(\mathrm{SD})$, or progressive disease (PD) according to the Response Evaluation Criteria in Solid Tumors version 1.1.

\section{Statistical analysis}

Statistical analyses were carried out with Statistical Product and Service Solutions software version 24.0 (SPSS Inc., Chicago, IL, USA) and R Statistical Software version 3.2.0 (Foundation for Statistical Computing, Vienna, Austria). All analyses were two-tailed, and the significance level was specified as $\mathrm{p}<0.05$. The primary objective of this study was to compare the OS of patients with $\mathrm{mNPC}$ treated with RT with or without concurrent 
chemotherapy. OS was defined as the time from metastasis to death from any cause or censored at the last visit or the final follow-up date of December 31, 2019. The secondary objective was progression-free survival (PFS), which was defined as the time from metastasis to disease progression or death from any cause or censored at the last visit or the final follow-up date of December 31, 2019. More specifically, locoregional progression-free survival (LRPFS) and distant progression-free survival (DPFS) were analyzed. The chi-square test or Fisher's exact test was used to compare categorical variables between two groups. Then, the baseline characteristics with significant differences were introduced into the logistic regression model to compute a propensity score for every patient. Propensity score matching (PSM) was employed to match patients with or without concurrent chemotherapy using the 1:1 nearest neighbor technique with a caliper of 0.1 to ensure a relatively good balance. Survival curves were evaluated using the Kaplan-Meier method with the log-rank test. Cox proportional hazards modeling was used to determine prognostic factors for OS. Multivariate analysis via stepwise selection was conducted including variables with $\mathrm{P}<0.1$ in univariate analyses. Exploratory subgroup analyses and landmark analyses were applied to the matched cohort to address bias from patients with a poor prognosis.

\section{Results}

\section{Baseline characteristics}

Among the 355 patients included in this study, 163 (45.9\%) received CCRT, while 192 (54.1\%) received RT alone. Patients and treatment characteristics before matching are summarized in Table 1 . Age was divided into two groups by a median age of 45 years old. The use of CCRT increased as the year of diagnosis increased and was associated with T4 disease and the application of IMRT. Before RT, most patients received platinum-based chemotherapy, such as PF, TP, or TPF, and approximately $70 \%$ of them underwent $4-6$ cycles. The median cycle number of PCT was 5 cycles in the PCT + CCRT group and 6 cycles in the PCT $+\mathrm{RT}$ group. The tumor response to PCT was satisfactory, and the most common response was PR. Even though patients received PCT, RT was still tolerated, as most patients had a Karnofsky performance score (KPS) rating of 90 . The RT dose for more than $90 \%$ of the patients reached 60 Gy and their median doses were both $70 \mathrm{~Gy}$. In addition to PCT and RT, 125 patients underwent local treatment at metastatic sites. Propensity score analysis was performed with the significant variables, including diagnosis year, $\mathrm{T}$ category, $\mathrm{RT}$ technique and RT dose, and all covariates were well balanced after matching with $\mathrm{P}>0.1$ (Table 1).

\section{Survival outcomes of CCRT after PCT}

The median follow-up time for the entire cohort was 50.8 months (95\% confidence interval [CI], 45.056.6 months). The addition of concurrent chemotherapy significantly improved survival outcomes, with a median OS time out of 60 months and a 5 -year OS rate of $53.0 \%$ compared to 40.1 months and $36.2 \%$, respectively, for $\mathrm{RT}$ alone $(\mathrm{P}=0.004)$ (Fig. 1A). In the matched cohort of 268 patients, similar results were observed, with median OS times out of 60 months vs 42.1 months and 5-year OS rates of $55.7 \%$ vs $39.0 \%$, respectively $(\mathrm{P}=0.034)$ (Fig. 1B). PFS was also analyzed in our matched cohort (Fig. 1C), but no significant difference between the two groups was observed (median PFS, 18.5 vs 23.7 months; $\mathrm{P}=0.083)$. However, the difference in DPFS between the $\mathrm{PCT}+\mathrm{CCRT}$ group and the PCT + RT group was close to being statistically significant (median DPFS, 29.4 vs 18.7 months; $\mathrm{P}=0.052$ ).

\section{Prognostic factors}

The results of the univariate and multivariate Cox regression models in the matched cohort were summarized in Table 2 . As only 13 patients had lymph node metastasis without organ metastasis, 11 patients had GP regimen, and 3 patients received RT with a maximum dose less than $60 \mathrm{~Gy}$, these patients were excluded in the analyses. On univariate analysis, the addition of CCRT to PCT, later diagnosis, 4-6 cycles of PCT before RT and local treatment at metastatic sites were associated with longer OS, while multiple liver metastases or multiple metastatic lesions were correlated with diminished OS. Ten variables with $\mathrm{P}<0.1$ in univariate analyses were entered into multivariate analysis, and PCT + CCRT remained independently associated with improved OS (HR, 0.59; 95\% CI, 0.39-0.88; $\mathrm{P}=0.009$ ). Additional favorable prognostic factors for OS included females and $\geq 4$ cycles of PCT. Multiple liver metastases and multiple metastatic lesions were still independent risk factors for OS.

\section{Combination of PCT and RT}

To determine whether the addition of concurrent chemotherapy could reduce the intensity of PCT before RT, we analyzed survival outcomes with different combinations of PCT and RT for the matching patients, showing the results in Fig. 2. Regardless of the PCT + RT group or PCT + CCRT group, the prognosis with 4-6 cycles of PCT was better than that with 1-3 cycles of PCT, among which 4-6 cycles of PCT followed by CCRT had excellent survival with a 5 -year OS rate of $60.4 \%$ (Fig. 2A). Therefore, enough cycles of PCT were necessary even with the addition of concurrent chemotherapy. Furthermore, we performed the analysis in patients with or without concurrent 
Table 1 Baseline characteristics

\begin{tabular}{|c|c|c|c|c|c|c|}
\hline & \multicolumn{2}{|c|}{ Before matching } & \multirow[b]{2}{*}{ P-value } & \multicolumn{2}{|l|}{ After matching } & \multirow[b]{2}{*}{ P-value } \\
\hline & $\begin{array}{l}P C T+R T \\
(N=192) \text { No. } \\
(\%)\end{array}$ & $\begin{array}{l}\mathrm{PCT}+\mathrm{CCRT} \\
(\mathrm{N}=163) \text { No. (\%) }\end{array}$ & & $\begin{array}{l}\mathrm{PCT}+\mathrm{RT} \\
(\mathrm{N}=134) \text { No. } \\
(\%)\end{array}$ & $\begin{array}{l}\mathrm{PCT}+\mathrm{CCRT} \\
(\mathrm{N}=134) \text { No. (\%) }\end{array}$ & \\
\hline \multicolumn{7}{|l|}{ Age (years) } \\
\hline$<45$ & $102(53.1)$ & $81(49.7)$ & 0.590 & $75(56.0)$ & $69(51.5)$ & 0.462 \\
\hline$\geq 45$ & $90(46.9)$ & $82(50.3)$ & & $59(44.0)$ & $65(48.5)$ & \\
\hline \multicolumn{7}{|l|}{ Sex } \\
\hline Male & $163(84.9)$ & $141(86.5)$ & 0.781 & $112(83.6)$ & $117(87.3)$ & 0.386 \\
\hline Female & $29(15.1)$ & $22(13.5)$ & & $22(16.4)$ & $17(12.7)$ & \\
\hline \multicolumn{7}{|l|}{ Diagnosis period } \\
\hline $2000-2005$ & $26(13.5)$ & $12(7.4)$ & 0.030 & $14(10.4)$ & $12(9.0)$ & 0.865 \\
\hline 2006-2011 & $66(34.4)$ & $45(27.6)$ & & 33 (24.6) & $36(26.9)$ & \\
\hline 2012-2017 & $100(52.1)$ & $106(65.0)$ & & $87(64.9)$ & $86(64.2)$ & \\
\hline \multicolumn{7}{|l|}{ Pathology } \\
\hline$|-| \mid$ & $22(11.5)$ & $13(8.0)$ & 0.358 & $12(9.0)$ & $10(7.5)$ & 0.656 \\
\hline III & $170(88.5)$ & $150(92.0)$ & & $122(91.0)$ & $124(92.5)$ & \\
\hline \multicolumn{7}{|l|}{ Tumor category } \\
\hline $\mathrm{T} 1$ & $7(3.6)$ & $8(4.9)$ & 0.012 & $6(4.5)$ & $2(1.5)$ & 0.539 \\
\hline $\mathrm{T} 2$ & $33(17.2)$ & $13(8.0)$ & & $15(11.2)$ & $13(9.7)$ & \\
\hline T3 & 95 (49.5) & $72(44.2)$ & & $65(48.5)$ & $67(50.0)$ & \\
\hline $\mathrm{T} 4$ & $57(29.7)$ & $70(42.9)$ & & $48(35.8)$ & $52(38.8)$ & \\
\hline \multicolumn{7}{|l|}{ Node category } \\
\hline NO & $6(3.1)$ & $5(3.1)$ & 0.830 & $3(2.2)$ & $5(3.7)$ & 0.231 \\
\hline N1 & $39(20.3)$ & $31(19.0)$ & & $24(17.9)$ & $28(20.9)$ & \\
\hline $\mathrm{N} 2$ & $79(41.1)$ & $75(46.0)$ & & $50(37.3)$ & $60(44.8)$ & \\
\hline N3 & $68(35.4)$ & $52(31.9)$ & & $57(42.5)$ & $41(30.6)$ & \\
\hline \multicolumn{7}{|l|}{ Bone metastasis } \\
\hline Absent & $47(24.5)$ & $49(30.1)$ & 0.150 & $34(25.4)$ & $41(30.6)$ & 0.605 \\
\hline Single & $56(29.2)$ & $55(33.7)$ & & $44(32.8)$ & $43(32.1)$ & \\
\hline Multiple & $89(46.4)$ & $59(36.2)$ & & $56(41.8)$ & $50(37.3)$ & \\
\hline \multicolumn{7}{|l|}{ Liver metastasis } \\
\hline Absent & $154(80.2)$ & $128(78.5)$ & 0.915 & $114(85.1)$ & $104(77.6)$ & 0.264 \\
\hline Single & $17(8.9)$ & $15(9.2)$ & & $8(6.0)$ & $14(10.4)$ & \\
\hline Multiple & $21(10.9)$ & $20(12.3)$ & & $12(9.0)$ & $16(11.9)$ & \\
\hline \multicolumn{7}{|l|}{ Lung metastasis } \\
\hline Absent & $165(85.9)$ & $128(78.5)$ & 0.148 & $108(80.6)$ & $104(77.6)$ & 0.832 \\
\hline Single & $10(5.2)$ & $16(9.8)$ & & $11(8.2)$ & $13(9.7)$ & \\
\hline Multiple & $17(8.9)$ & $19(11.7)$ & & $15(11.2)$ & $17(12.7)$ & \\
\hline \multicolumn{7}{|c|}{ No. of metastatic organs } \\
\hline None & $10(5.2)$ & $7(4.3)$ & 0.441 & $8(6.0)$ & $5(3.7)$ & 0.375 \\
\hline Single organ & $157(81.8)$ & $127(77.9)$ & & $109(81.3)$ & $105(78.4)$ & \\
\hline Multiple organs & $25(13.0)$ & $29(17.8)$ & & $17(12.7)$ & 24 (17.9) & \\
\hline \multicolumn{7}{|c|}{ Sites of organ metastasis } \\
\hline Absent & $10(5.2)$ & $7(4.3)$ & 0.226 & $8(6.0)$ & $5(3.7)$ & 0.527 \\
\hline Single & $62(32.3)$ & $67(41.1)$ & & $47(35.1)$ & $54(40.3)$ & \\
\hline Multiple & $120(62.5)$ & $89(54.6)$ & & $79(59.0)$ & $75(56.0)$ & \\
\hline \multicolumn{7}{|c|}{ Distant nodal metastasis } \\
\hline Absent & $170(88.5)$ & $141(86.5)$ & 0.806 & $116(86.6)$ & $115(85.8)$ & 1.000 \\
\hline Single region & $19(9.9)$ & $19(11.7)$ & & $16(11.9)$ & $16(11.9)$ & \\
\hline Multiple regions & $3(1.6)$ & $3(1.8)$ & & $2(1.5)$ & $3(2.2)$ & \\
\hline
\end{tabular}


Table 1 (continued)

\begin{tabular}{|c|c|c|c|c|c|c|}
\hline & \multicolumn{2}{|c|}{ Before matching } & \multirow[b]{2}{*}{ P-value } & \multicolumn{2}{|c|}{ After matching } & \multirow[b]{2}{*}{ P-value } \\
\hline & $\begin{array}{l}\mathrm{PCT}+\mathrm{RT} \\
(\mathrm{N}=192) \text { No. } \\
(\%)\end{array}$ & $\begin{array}{l}\mathrm{PCT}+\mathrm{CCRT} \\
(\mathrm{N}=163) \text { No. (\%) }\end{array}$ & & $\begin{array}{l}\mathrm{PCT}+\mathrm{RT} \\
(\mathrm{N}=134) \text { No. } \\
(\%)\end{array}$ & $\begin{array}{l}\mathrm{PCT}+\mathrm{CCRT} \\
(\mathrm{N}=134) \text { No. }(\%)\end{array}$ & \\
\hline \multicolumn{7}{|l|}{ Metastatic type } \\
\hline Distant metastasis without LN involvement & $170(88.5)$ & $141(86.5)$ & 0.547 & $116(86.6)$ & $115(85.8)$ & 0.506 \\
\hline Only distant LN metastasis & $10(5.2)$ & $7(4.3)$ & & $8(6.0)$ & $5(3.7)$ & \\
\hline Distant metastasis with LN involvement & $12(6.2)$ & $15(9.2)$ & & $10(7.5)$ & $14(10.4)$ & \\
\hline \multicolumn{7}{|l|}{ No. of metastatic lesions } \\
\hline Oligo & $60(31.2)$ & $68(41.7)$ & 0.053 & $45(33.6)$ & $53(39.6)$ & 0.310 \\
\hline Multiple & $132(68.8)$ & $95(58.3)$ & & $89(66.4)$ & $81(60.4)$ & \\
\hline \multicolumn{7}{|l|}{ PCT regimen } \\
\hline PF & $69(35.9)$ & $43(26.4)$ & 0.052 & $48(35.8)$ & $36(26.9)$ & 0.157 \\
\hline GP & $12(6.2)$ & $4(2.5)$ & & $7(5.2)$ & $4(3.0)$ & \\
\hline $\mathrm{TP}$ & $46(24.0)$ & $39(23.9)$ & & $35(26.1)$ & $31(23.1)$ & \\
\hline TPF & $51(26.6)$ & $59(36.2)$ & & $36(26.9)$ & 48 (35.8) & \\
\hline Others & $14(7.3)$ & $18(11.0)$ & & $8(6.0)$ & $15(11.2)$ & \\
\hline \multicolumn{7}{|l|}{ No. of PCT cycles } \\
\hline $1-3$ & 35 (18.2) & $41(25.2)$ & 0.232 & $23(17.2)$ & $33(24.6)$ & 0.317 \\
\hline $4-6$ & $142(74.0)$ & $113(69.3)$ & & 103 (76.9) & $93(69.4)$ & \\
\hline$>6$ & $15(7.8)$ & $9(5.5)$ & & $8(6.0)$ & $8(6.0)$ & \\
\hline \multicolumn{7}{|l|}{ Response to PCT } \\
\hline$C R$ & $6(3.1)$ & $3(1.8)$ & 0.214 & $5(3.7)$ & $3(1.8)$ & 0.282 \\
\hline PR & $123(64.1)$ & $96(58.9)$ & & $86(64.2)$ & 78 (58.2) & \\
\hline SD & $18(9.4)$ & $27(16.6)$ & & $12(9.0)$ & $22(16.4)$ & \\
\hline Unknown & $45(23.4)$ & $37(22.7)$ & & $31(23.1)$ & $31(23.1)$ & \\
\hline \multicolumn{7}{|l|}{ KPS before RT } \\
\hline 90 & $159(82.8)$ & $143(87.7)$ & 0.147 & $113(84.3)$ & $118(88.1)$ & 0.227 \\
\hline 80 & $9(4.7)$ & $10(6.1)$ & & $7(5.2)$ & $9(6.7)$ & \\
\hline 70 & $2(1.0)$ & $2(1.2)$ & & $1(0.7)$ & $2(1.5)$ & \\
\hline Unknown & $22(11.5)$ & $8(4.9)$ & & $13(9.7)$ & $5(3.7)$ & \\
\hline \multicolumn{7}{|l|}{ Technique of RT } \\
\hline Conventional RT & $67(34.9)$ & $28(17.2)$ & $<0.001$ & 29 (21.6) & $28(17.2)$ & 1.000 \\
\hline IMRT & $113(58.9)$ & $131(80.4)$ & & 104 (77.6) & 104 (77.6) & \\
\hline Unknown & $12(6.2)$ & $4(2.5)$ & & $1(0.7)$ & $2(1.5)$ & \\
\hline \multicolumn{7}{|l|}{ RT dose (Gy) } \\
\hline$<60$ & $11(5.7)$ & $2(1.2)$ & 0.020 & $1(0.7)$ & $2(1.5)$ & 1.000 \\
\hline$\geq 60$ & $179(93.2)$ & $161(98.8)$ & & 133 (99.3) & $132(98.5)$ & \\
\hline Unknown & $2(1.0)$ & $0(0.0)$ & & $0(0.0)$ & $0(0.0)$ & \\
\hline \multicolumn{7}{|l|}{ Local treatment of metastatic sites } \\
\hline No & $128(66.7)$ & $102(62.6)$ & 0.489 & $81(60.4)$ & $86(64.2)$ & 0.529 \\
\hline Yes & $64(33.3)$ & $61(37.4)$ & & $53(39.6)$ & $48(35.8)$ & \\
\hline
\end{tabular}

$P C T$ palliative chemotherapy, $R T$ radiotherapy, $C C R T$ concurrent chemoradiotherapy, No. number, $L N$ lymph node, $P F$ cisplatin plus 5-fluorouracil, GP gemcitabine plus cisplatin, TP cisplatin plus docetaxel, TPF cisplatin plus docetaxel plus 5-fluorouracil, $C R$ complete response, $P R$ partial response, $S D$ stable disease, KPS Karnofsky performance score, IMRT intensity-modulated radiotherapy

single-agent platinum such as cisplatin or platinumcombination chemotherapy such as PF. We identified that concurrent single-agent platinum was more appropriate during RT in patients with PCT (Fig. 2B).
A trend toward improved OS was observed for patients receiving concurrent single-agent platinum after 4-6 cycles of PCT $(\mathrm{P}=0.074)$ (Fig. $2 \mathrm{C})$. 

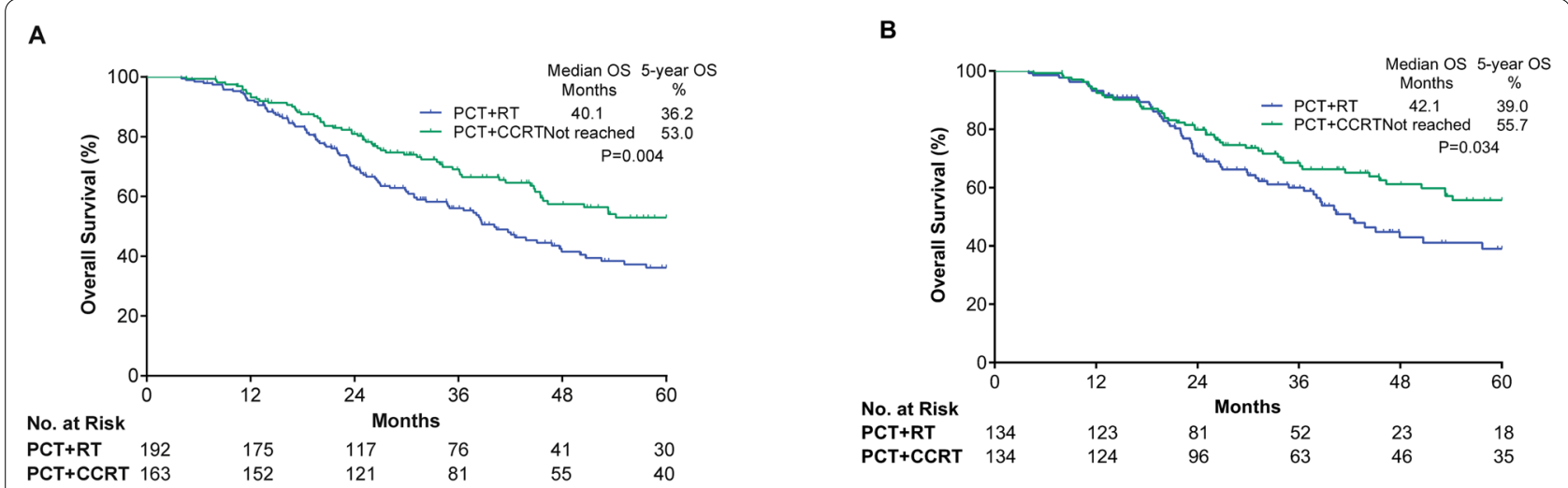

C

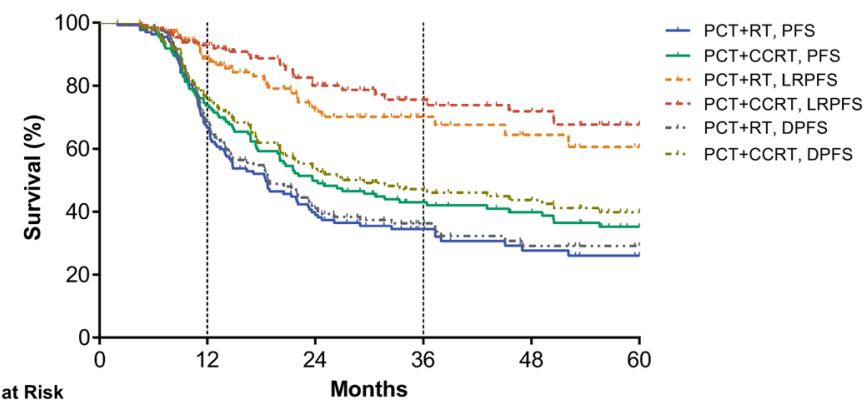

\begin{tabular}{|c|c|c|c|c|c|c|c|}
\hline \multirow{2}{*}{$\begin{array}{l}\text { No. at Risk } \\
\text { PCT+RT }\end{array}$} & \multicolumn{7}{|c|}{ Months } \\
\hline & 134 & & 38 & 47 & 29 & 17 & 13 \\
\hline \multirow[t]{3}{*}{ PCT+CCRT } & 134 & & 98 & 63 & 44 & 36 & 25 \\
\hline & \multicolumn{3}{|c|}{$\mathrm{PCT}+\mathrm{RT}$} & \multicolumn{3}{|c|}{ PCT+CCRT } & P-value \\
\hline & $\begin{array}{c}1 \text {-year } \\
\%\end{array}$ & $\begin{array}{c}\text { 3-year } \\
\%\end{array}$ & $\begin{array}{l}\text { Median } \\
\text { months }\end{array}$ & $\begin{array}{c}1 \text {-year } \\
\%\end{array}$ & $\begin{array}{c}\text { 3-year } \\
\%\end{array}$ & $\begin{array}{l}\text { Median } \\
\text { months }\end{array}$ & $\begin{array}{l}\text { (PCT+RT vs. } \\
\text { PCT+CCRT) }\end{array}$ \\
\hline PFS & 65.9 & 34.5 & 18.5 & 73.0 & 43.0 & 23.7 & 0.083 \\
\hline LRPFS & 88.7 & 70.1 & Not reached & 92.8 & 75.6 & Not reached & 0.252 \\
\hline DPFS & 67.6 & 36.2 & 18.7 & 75.4 & 47.2 & 29.4 & 0.052 \\
\hline
\end{tabular}

Fig. 1 Survival outcomes for patients treated with CCRT or RT after PCT. A OS for all patients. B OS for propensity score-matched patients. C PFS, including LRPFS and DPFS for propensity score-matched patients. CCRT concurrent chemoradiotherapy, $R T$ radiotherapy, $P C T$ palliative chemotherapy, OS overall survival, PFS progression-free survival, LRPFS locoregional progression-free survival, DPFS distant progression-free survival

\section{Subgroup and landmark analyses}

Subgroup analyses of clinical factors were shown in Fig. 3. The absence of bone metastasis, the presence of liver metastasis and a solitary metastasis derived more benefit from PCT followed by CCRT.

The results of landmark analysis, which evaluated the impact of CCRT after PCT on long-term survival, were presented in Fig. 4. Among the patients who survived for $\geq 1$ year after diagnosis, PCT followed by CCRT remained associated with improved OS $(\mathrm{P}=0.028)$. However, this survival difference was not significant in the patients who survived for $\geq 2$ years $(\mathrm{P}=0.122)$.

\section{Discussion}

Benefits from the treatment of primary tumors in metastatic disease have been demonstrated for multiple tumor types, such as renal cancer, breast cancer and NPC [12, 13]. The OS of patients with $\mathrm{mNPC}$ who received RT was prolonged in our study (Additional file 1: Fig. S2), consistent with the results of previous studies. Although platinum-based chemotherapy remains the foundation of mNPC treatment, the phase III trial comparing the survival of patients with or without RT in endemic regions confirmed that radiotherapy added to chemotherapy significantly improved OS in chemotherapy-sensitive patients with mNPC (2-year OS, $76.4 \%$ vs $54.5 \%$; $\mathrm{P}=0.004)$, providing more convincing evidence [11]. However, the long-term efficacy of the trial has not yet been reported, and the 5-year OS rate of RT after PCT in previous retrospective studies had heterogeneous results varying from $16.6 \%$ to $34 \%$, which was disappointing when compared with the 5-year OS of approximately $80 \%$ for non-metastatic NPC (Additional file 1: Table S2) $[9,10,14-16]$. What else can we do for mNPC except for the addition of RT? The purpose of this study was to provide a useful strategy for clinicians in treating $\mathrm{mNPC}$ 
Table 2 Univariate and multivariate Cox regression models in the propensity score-matched cohort

\begin{tabular}{|c|c|c|c|c|}
\hline & \multicolumn{2}{|l|}{ Univariate } & \multirow{2}{*}{$\begin{array}{l}\text { Multivariate } \\
\text { HR }(95 \% \mathrm{Cl})\end{array}$} & \multirow[b]{2}{*}{ P-value } \\
\hline & $\mathrm{HR}(95 \% \mathrm{Cl})$ & P-value & & \\
\hline \multicolumn{5}{|l|}{ Group } \\
\hline$P C T+R T$ vs. PCT + CCRT & $0.67(0.46-0.97)$ & 0.036 & $0.59(0.39-0.88)$ & 0.009 \\
\hline \multicolumn{5}{|l|}{ Age (years) } \\
\hline$<45$ vs. $\geq 45$ & $1.18(0.81-1.72)$ & 0.389 & & \\
\hline \multicolumn{5}{|l|}{ Sex } \\
\hline Male vs. Female & $0.53(0.28-1.00)$ & 0.054 & $0.50(0.27-0.94)$ & 0.033 \\
\hline \multicolumn{5}{|l|}{ Diagnosis period } \\
\hline 2000-2005 vs. 2006-2011 & $0.52(0.30-0.92)$ & 0.025 & & \\
\hline 2000-2005 vs. 2012-2017 & $0.46(0.27-0.77)$ & 0.003 & & \\
\hline \multicolumn{5}{|l|}{ Pathology } \\
\hline |-I| vs. III & $0.78(0.58-1.04)$ & 0.093 & & \\
\hline \multicolumn{5}{|l|}{ Tumor category } \\
\hline T1-2 vs. T3-4 & $1.06(0.61-1.83)$ & 0.834 & & \\
\hline \multicolumn{5}{|l|}{ Node category } \\
\hline No-1 vs. N2-3 & $1.10(0.71-1.71)$ & 0.670 & & \\
\hline \multicolumn{5}{|l|}{ Bone metastasis } \\
\hline Absent vs. Single & $0.66(0.39-1.11)$ & 0.117 & & \\
\hline Absent vs. Multiple & $1.77(0.87-2.12)$ & 0.177 & & \\
\hline \multicolumn{5}{|l|}{ Liver metastasis } \\
\hline Absent vs. Single & $0.83(0.40-1.71)$ & 0.606 & $1.06(0.51-2.22)$ & 0.871 \\
\hline Absent vs. Multiple & $2.32(1.38-3.88)$ & 0.001 & $1.95(1.13-3.35)$ & 0.016 \\
\hline \multicolumn{5}{|l|}{ Lung metastasis } \\
\hline Absent vs. Single & $0.79(0.38-1.62)$ & 0.514 & & \\
\hline Absent vs. Multiple & $1.32(0.74-2.37)$ & 0.346 & & \\
\hline \multicolumn{5}{|l|}{ Distant nodal metastasis } \\
\hline Absent vs. Present & $1.53(0.92-2.55)$ & 0.100 & & \\
\hline \multicolumn{5}{|l|}{ No. of metastatic organs } \\
\hline Single organ vs. Multiple organs & $1.62(0.98-2.66)$ & 0.059 & & \\
\hline \multicolumn{5}{|l|}{ Metastatic situation } \\
\hline $\begin{array}{l}\text { Organ metastasis without } L N \text { involvement vs. Organ } \\
\text { metastasis with } L N \text { involvement }\end{array}$ & $1.26(0.94-1.69)$ & 0.116 & & \\
\hline \multicolumn{5}{|l|}{ No. of metastatic lesions } \\
\hline Oligo vs. Multiple & $2.18(1.42-3.34)$ & $<0.001$ & $1.97(1.23-3.16)$ & 0.005 \\
\hline \multicolumn{5}{|l|}{$\mathrm{PCT}$ regimen } \\
\hline PFvs.TP & $1.21(0.77-1.91)$ & 0.415 & & \\
\hline PF vs. TPF & $0.65(0.40-1.05)$ & 0.077 & & \\
\hline \multicolumn{5}{|l|}{ No. of PCT cycles } \\
\hline $1-3$ vs. $4-6$ & $0.61(0.40-0.92)$ & 0.020 & $0.49(0.31-0.77)$ & 0.002 \\
\hline $1-3$ vs. $>6$ & $0.47(0.18-1.22)$ & 0.120 & $0.27(0.09-0.77)$ & 0.014 \\
\hline \multicolumn{5}{|l|}{ Response to PCT } \\
\hline CR/PR vs. SD & $1.19(0.93-1.53)$ & 0.159 & & \\
\hline \multicolumn{5}{|l|}{ KPS before RT } \\
\hline 90 vs. $70-80$ & $1.30(0.69-2.42)$ & 0.417 & & \\
\hline \multicolumn{5}{|l|}{ Technique of RT } \\
\hline Conventional RT vs. IMRT & $0.74(0.49-1.13)$ & 0.162 & & \\
\hline \multicolumn{5}{|l|}{ Local treatment of metastatic sites } \\
\hline No vs. Yes & $0.65(0.44-0.98)$ & 0.040 & $0.69(0.45-1.05)$ & 0.082 \\
\hline
\end{tabular}

$P C T$ palliative chemotherapy, $R T$ radiotherapy, CCRT concurrent chemoradiotherapy, HR hazard ratio, Cl confidence interval, No. number, $L N$ lymph node, $P F$ cisplatin plus 5-fluorouracil, TP cisplatin plus docetaxel, TPF cisplatin plus docetaxel plus 5-fluorouracil, $C R$ complete response, $P R$ partial response, $S D$ stable disease, $K P S$ Karnofsky performance score, IMRT intensity-modulated radiotherapy 


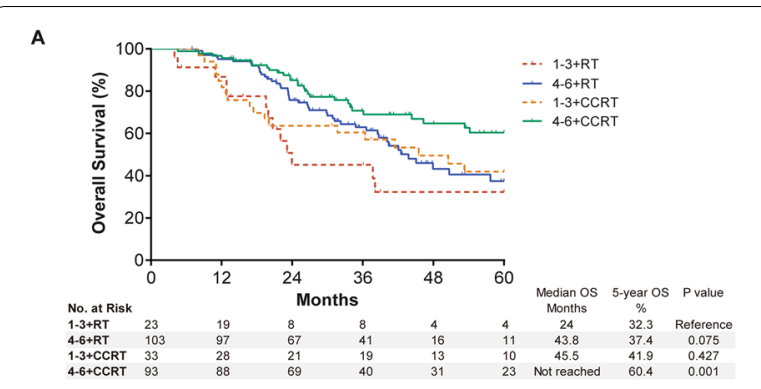

B

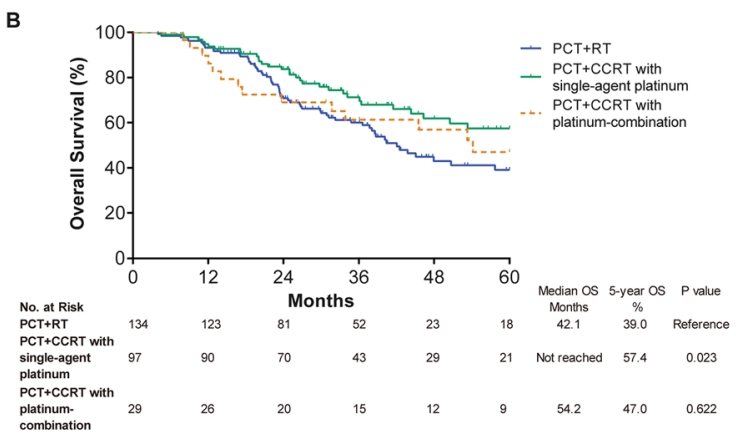

C

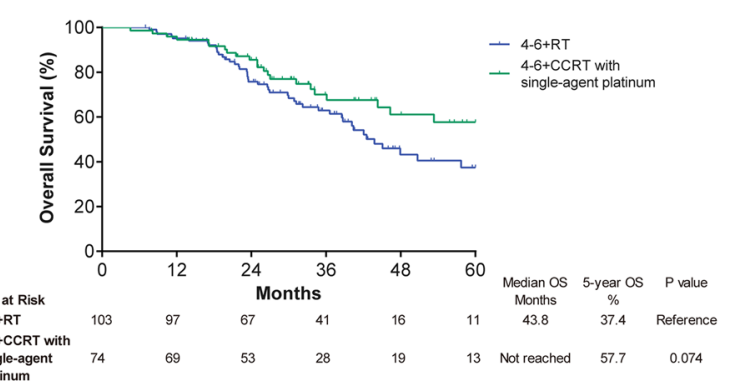

Fig. 2 OS for patients receiving different combinations of systemic and locoregional treatments after propensity score matching. A Different cycles of PCT. B Different regimens of concurrent chemotherapy. C Single-agent platinum versus radiotherapy alone after 4-6 cycles of PCT. OS overall survival, PCT palliative chemotherapy, CCRT concurrent chemoradiotherapy, $R T$ radiotherapy

with traditional therapies. To the best of our knowledge, this study is the first attempt to compare PCT followed by RT with or without concurrent chemotherapy.

In this study, we report the outcomes of continuous de novo metastatic NPC patients treated with PCT and RT at an institution in an endemic area for nearly 20 years. The addition of chemotherapy during RT significantly improved OS compared with RT alone when finishing PCT. An absolute improvement in the 5-year OS rate of approximately $16.8 \%$ was identified for the entire cohort. After addressing selection limitations related to the use of PSM, the advantage of CCRT could still not be ignored. The best time to perform CCRT was suggested to be after 4-6 cycles of PCT, and single-agent platinum therapeutics such as cisplatin can be of great benefit to patients.

Concurrent chemoradiotherapy has been recommended for patients with locoregionally advanced NPC [17]. A phase II randomized controlled trial demonstrated that concurrent weekly cisplatin chemotherapy could improve the OS of patients with locally recurrent NPC, especially those with disease classified in an advanced $\mathrm{T}$ category. The difference in grade 3-4 toxicity between CCRT and RT alone was not significant [18]. In other metastatic tumor types, CCRT was reported to improve survival outcomes [19-22]. In our study, CCRT was recommended as survival was significantly improved while most grade 3 to 4 toxic effects were not significantly increased (Additional file 1: Table S3). We believe that concurrent chemotherapy enhances tumor control, acts as a radiotherapy sensitizer and eradicates micrometastases, leading to an additive or synergistic effect on tumor killing. Additionally, control of the primary tumor prevents further self-seeding of metastases [23], and the abscopal effect of radiotherapy induces regression at nonirradiated, distant tumor sites [24].

The details for chemotherapy before or during RT, such as agents and cycle numbers, are inconclusive due to limited medical records [9]. Some evidence indicated that there was no significant difference between patients who received at least six cycles of chemotherapy and those who received less than six cycles [25]. Chemoresistance may occur as the number of cycles increases. However, progression may appear with less intense chemotherapy, especially in patients who undergo fewer than four cycles $[6,7]$. After patients receive $4-6$ cycles of chemotherapy, chemoradiotherapy should be applied. A single agent for concurrent chemotherapy, such as cisplatin, may be the best choice because it has less severe side effects, unlike doublet or multiple agents, and much evidence has demonstrated the important role of cisplatin in concurrent chemotherapy [26].

The prognostic characteristics of mNPC have been explored in many studies. A number of these studies have indicated that patients with limited metastatic lesions had more favorable outcomes than those with liver metastasis or multiple metastatic lesions [27, 28]. For patients with favorable outcomes, consolidated therapy of the primary tumor may make metastatic disease a curative disease, while actively curing the primary disease may prolong local control for patients with unfavorable outcomes. However, identifying candidates who are most likely to benefit from CCRT needs further research. Relatively good outcomes have been observed for patients receiving $\mathrm{RT}$ if they were sensitive to $\mathrm{PCT}$ or if post-PCT 


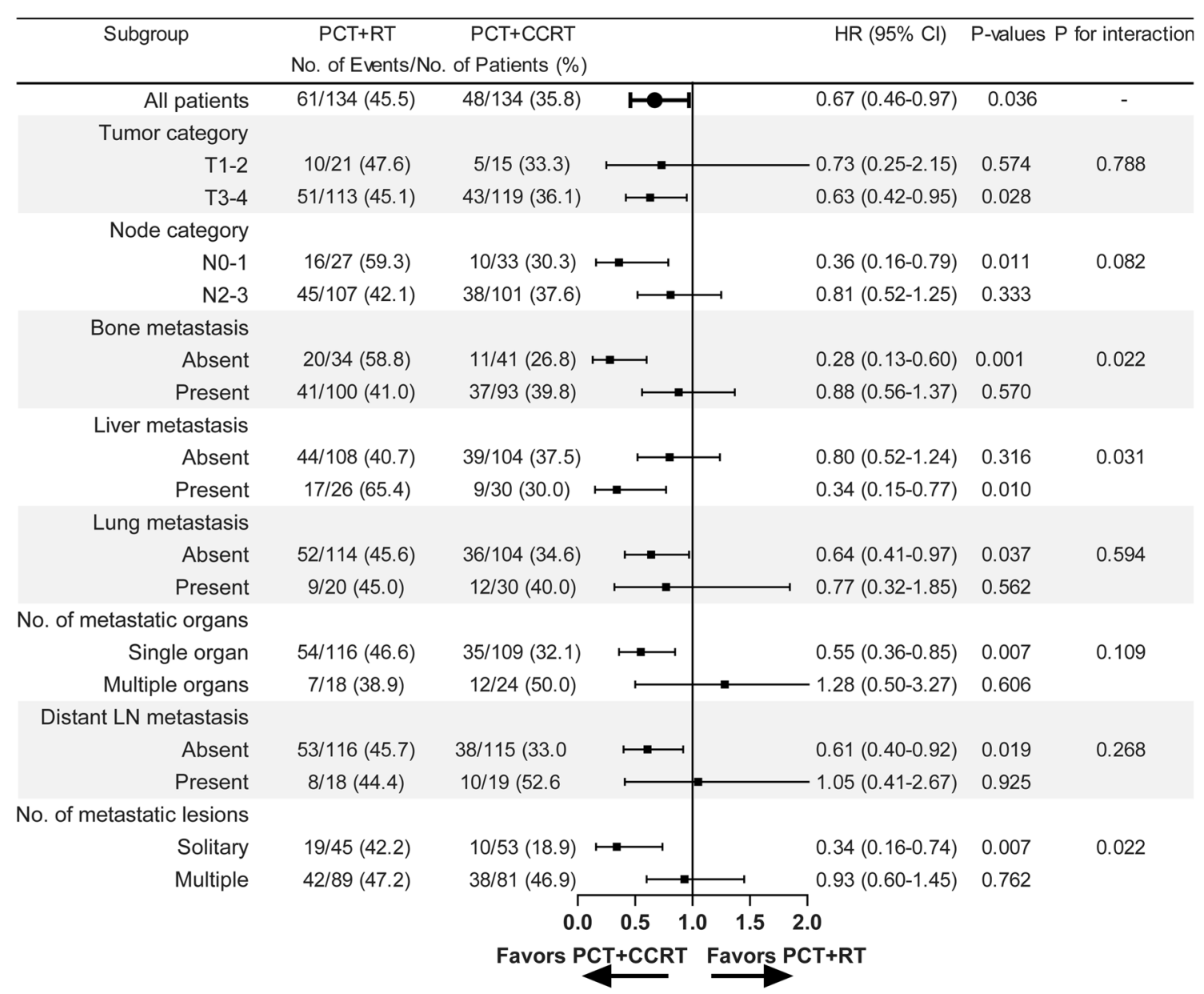

Fig. 3 Forest plot of CCRT versus RT after PCT by subgroups for propensity score-matched patients. CCRT concurrent chemoradiotherapy, $R T$ radiotherapy, $P C T$ palliative chemotherapy, HR hazard ratio, Cl confidence interval, No. number, LN lymph node
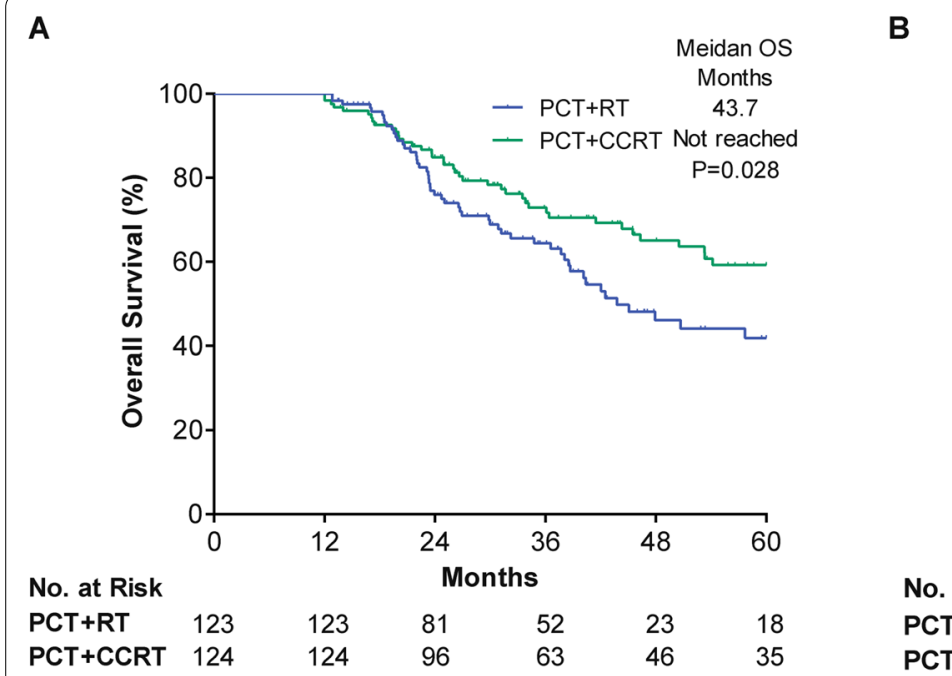

No. at Risk

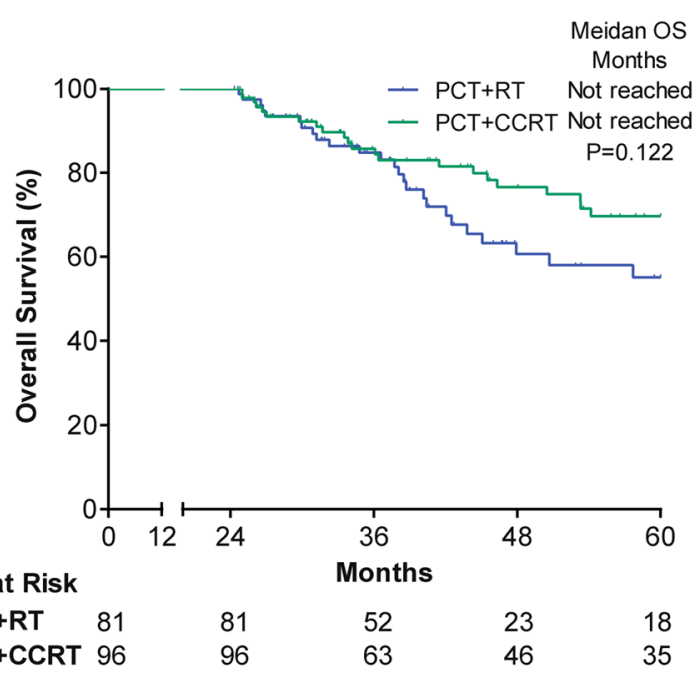

Fig. 4 Landmark analysis of OS for propensity score-matched patients receiving PCT followed by CCRT or RT. A Patients survived for $\geq 1$ year. B Patients survived for $\geq 2$ years. OS overall survival; PCT palliative chemotherapy, CCRT concurrent chemoradiotherapy, RT radiotherapy 
Epstein-Barr virus (EBV) levels decreased [29, 30]. Perhaps it helps when selecting patients to deliver CCRT.

Currently, there is an exciting era of developing immune checkpoint inhibitors in NPC [31]. A phase I clinical trial of 27 patients with recurrent or metastatic NPC suggested that pembrolizumab treatment resulted in a median OS of 16.5 months. [32] Another phase II trial of nivolumab also suggested the potential use of immunotherapy for $\mathrm{mNPC}$ as the median OS of 44 patients receiving nivolumab was 17.1 months. [33] In addition to monotherapy, a phase I clinical trial from China demonstrated that the therapeutic effect of camrelizumab combined with chemotherapy was superior to that of camrelizumab alone [34]. The superior efficacy of the camrelizumab combination was recently confirmed in a phase III randomized study [35]. Therefore, how to use immunotherapy in addition to chemotherapy and radiotherapy to maximize the survival of patients with $\mathrm{mNPC}$ is worth further effort.

Our study had several limitations that should be mentioned. The source of patients who underwent PCT followed by RT was restricted to one hospital, and the sample size was not sufficiently large. As this study was retrospective in nature, selection bias and imbalances existed. Plasma EBV testing results were not available, although EBV was an important factor for therapeutic monitoring and prognostic evaluations. In addition, quality of life, late toxicity and some details on the following lines of therapy were not considered in this study. Thus, prospective studies are warranted to support our findings.

\section{Conclusions}

The real-world study suggests that concurrent chemoradiotherapy significantly improves OS compared with radiotherapy alone after palliative chemotherapy in patients with de novo metastatic nasopharyngeal carcinoma. More specifically, concurrent chemoradiotherapy with single-agent platinum after 4-6 cycles of chemotherapy can be considered.

\footnotetext{
Abbreviations

NPC: Nasopharyngeal carcinoma; mNPC: De novo metastatic nasopharyngeal carcinoma; NCCN: National Comprehensive Cancer Network; PCT: Palliative chemotherapy; RT: Radiotherapy; OS: Overall survival; CCRT: Concurrent chemoradiotherapy; HR: Hazard ratio; WHO: World Health Organization; PF: Cisplatin plus 5-fluorouracil; TP: Cisplatin plus docetaxel; TPF: Cisplatin plus docetaxel plus 5-fluorouracil; IMRT: Intensity-modulated radiotherapy; CR: Complete response; PR: Partial response; SD: Stable disease; PD: Progressive disease; PFS: Progression-free survival; LRPFS: Locoregional progression-free survival; DPFS: Distant progression-free survival; PSM: Propensity score matching; Cl: Confidence interval; KPS: Karnofsky performance score; EBV: Epstein-Barr virus.
}

\section{Supplementary Information}

The online version contains supplementary material available at https://doi. org/10.1186/s12935-022-02464-7.

Additional file 1: Description of the radiotherapy. Figure S1. Patient selection diagram. Figure S2. Overall Survival (OS) for patients treated with chemoradiotherapy (CRT) or palliative chemotherapy (PCT) in de novo metastatic nasopharyngeal carcinoma. Table S1. Details of common chemotherapy regimens. Table S2. Summary of studies related to locoregional radiotherapy in de novo metastatic nasopharyngeal carcinoma. Table S3. Adverse effects. References.

Acknowledgements

Not applicable.

\section{Authors' contributions}

Conception and design: SHZ, YTW and YFX; Administrative support: SRL, CC and YFX; Provision of study materials or patients: SRL and YFX; Collection and assembly of data: SHZ, YTW, ZLH, GNW, JTLand SRD; Data analysis and interpretation: SHZ, CC and YFX; Manuscript writing: All authors. All authors read and approved the final manuscript.

\section{Funding}

This work was supported by the National Key R \& D Program of Precise Medical Research of China (NO. 2016YFC0904600), the National Natural Science Foundation of China (No. 81872464), and the Natural Science Foundation of Guangdong Province (No. 2018A030310236).

\section{Availability of data and materials}

The authenticity of this article has been validated by uploading the key raw data onto the Research Data Deposit public platform (www.researchdata.org. $\mathrm{cn})$. All data will be shared upon request to the corresponding author.

\section{Declarations}

\section{Ethics approval and consent to participate}

The study was conducted in accordance with the Declaration of Helsinki (as revised in 2013). The study was approved by ethics board of Sun Yat-sen University Cancer Center and individual consent for this retrospective analysis was waived.

\section{Consent for publication}

Not applicable.

\section{Competing interests}

The authors declare that they have no competing interests.

\section{Author details}

${ }^{1}$ State Key Laboratory of Oncology in South China, Collaborative Innovation Center for Cancer Medicine, Guangdong Key Laboratory of Nasopharyngeal Carcinoma Diagnosis and Therapy, Sun Yat-Sen University Cancer Center, Guangzhou, China. ${ }^{2}$ Department of Radiation Oncology, Sun Yat-Sen University Cancer Center, Guangzhou, China. ${ }^{3}$ Zhongshan Medical College, Sun Yat-Sen University, Guangzhou, China. ${ }^{4}$ Department of Pathology, Sun Yat-Sen University Cancer Center, Guangzhou, China. ${ }^{5}$ Department of Head \& Neck Surgery, Sun Yat-Sen University Cancer Center, Guangzhou, China.

Received: 26 July 2021 Accepted: 11 January 2022

Published online: 24 January 2022 


\section{References}

1. Chen Y, Chan ATC, Le Q, et al. Nasopharyngeal carcinoma. Lancet. 2019;394(10192):64-80.

2. Wei KR, Zheng RS, Zhang SW, et al. Nasopharyngeal carcinoma incidence and mortality in China, 2013. Chin J Cancer. 2017;36(1):90

3. Lee AW, Poon YF, Foo W, et al. Retrospective analysis of 5037 patients with nasopharyngeal carcinoma treated during 1976-1985: overall survival and patterns of failure. Int J Radiat Oncol Biol Phys. 1992;23(2):261-70.

4. Liao W, Tian M, Chen N. Characteristic and novel therapeutic strategies of nasopharyngeal carcinoma with synchronous metastasis. Cancer Manag Res. 2019;11:8431-42.

5. Head and Neck Cancers. NCCN clinical practice guidelines in oncology. Version 3.2021. https://www.nccn.org/professionals/physician_gls/pdf/ head-and-neck.pdf. Accessed 27 Apr 2021.

6. Tian YH, Zou WH, Xiao WW, et al. Oligometastases in AJCC stage IVc nasopharyngeal carcinoma: A subset with better overall survival. Head Neck. 2016;38(8):1152-7.

7. Zeng L, Tian YM, Huang Y, et al. Retrospective analysis of 234 nasopharyngeal carcinoma patients with distant metastasis at initial diagnosis: therapeutic approaches and prognostic factors. PLoS ONE. 2014;9(9):e108070.

8. Chen M, Jiang R, Guo L, et al. Locoregional radiotherapy in patients with distant metastases of nasopharyngeal carcinoma at diagnosis. Chin J Cancer. 2013;32(11):604-13.

9. Rusthoven CG, Lanning RM, Jones BL, et al. Metastatic nasopharyngeal carcinoma: Patterns of care and survival for patients receiving chemotherapy with and without local radiotherapy. Radiother Oncol. 2017:124(1):139-46.

10. Verma $V$, Allen PK, Simone $C N$, et al. Addition of definitive radiotherapy to chemotherapy in patients with newly diagnosed metastatic nasopharyngeal cancer. J Natl Compr Canc Netw. 2017;15(11):1383-91.

11. You R, Liu YP, Huang PY, et al. Efficacy and safety of locoregional radiotherapy with chemotherapy vs chemotherapy alone in de novo metastatic nasopharyngeal carcinoma. JAMA Oncol. 2020;6(9):1345-52.

12. Soran A, Ozmen V, Ozbas S, et al. Randomized trial comparing resection of primary tumor with no surgery in stage IV breast cancer at presentation: protocol MF07-01. Ann Surg Oncol. 2018;25(11):3141-9.

13. Mickisch GH, Garin A, van Poppel H, et al. Radical nephrectomy plus interferon-alfa-based immunotherapy compared with interferon alfa alone in metastatic renal-cell carcinoma: a randomised trial. Lancet. 2001;358(9286):966-70.

14. Lin SJ, Tham IWK, Pan JJ, et al. Combined high-dose radiation therapy and systemic chemotherapy improves survival in patients with newly diagnosed metastatic nasopharyngeal cancer. Am J Clin Oncol. 2012;35(5):474-9.

15. Hu SX, He XH, Dong M, et al. Systemic chemotherapy followed by locoregional definitive intensity-modulated radiation therapy yields prolonged survival in nasopharyngeal carcinoma patients with distant metastasis at initial diagnosis. Med Oncol. 2015. https://doi.org/10.1007/ s12032-015-0663-2.

16. Wang BC, Cao RB, Fu C, et al. The efficacy and safety of PD-1/PD-L1 inhibitors in patients with recurrent or metastatic nasopharyngeal carcinoma: A systematic review and meta-analysis. Oral Oncol. 2020;104:104640.

17. Lin JC, Jan JS, Hsu CY, et al. Phase III study of concurrent chemoradiotherapy versus radiotherapy alone for advanced nasopharyngeal carcinoma: positive effect on overall and progression-free survival. J Clin Oncol. 2003;21(4):631-7.

18. Guan Y, Liu S, Wang H, et al. Long-term outcomes of a phase II randomized controlled trial comparing intensity-modulated radiotherapy with or without weekly cisplatin for the treatment of locally recurrent nasopharyngeal carcinoma. Chin J Cancer. 2016. https://doi.org/10.1186/ s40880-016-0081-7.

19. Crane $\mathrm{CH}$, Janjan NA, Abbruzzese JL, et al. Effective pelvic symptom control using initial chemoradiation without colostomy in metastatic rectal cancer. Int J Radiat Oncol Biol Phys. 2001;49(1):107-16.

20. Kim MM, Rana V, Janjan NA, et al. Clinical benefit of palliative radiation therapy in advanced gastric cancer. Acta Oncol. 2008;47(3):421-7.

21. Bae SH, Park W, Choi DH, et al. Palliative radiotherapy in patients with a symptomatic pelvic mass of metastatic colorectal cancer. Radiat Oncol. 2011;6(1):52-52.
22. Wang Z, Ren ZG, Ma NY, et al. Intensity modulated radiotherapy for locally advanced and metastatic pancreatic cancer: a mono-institutional retrospective analysis. Radiat Oncol. 2015;10(1):14.

23. Comen E, Norton L, Massague J. Clinical implications of cancer selfseeding. Nat Rev Clin Oncol. 2011;8(6):369-77.

24. Reynders K, Illidge T, Siva S, et al. The abscopal effect of local radiotherapy: using immunotherapy to make a rare event clinically relevant. Cancer Treat Rev. 2015;41(6):503-10.

25. Jin $Y$, Shi $Y X$, Cai $X Y$, et al. Comparison of five cisplatin-based regimens frequently used as the first-line protocols in metastatic nasopharyngeal carcinoma. J Cancer Res Clin Oncol. 2012;138(10):1717-25.

26. Chan AT, Leung SF, Ngan RK, et al. Overall survival after concurrent cisplatin-radiotherapy compared with radiotherapy alone in locoregionally advanced nasopharyngeal carcinoma. J Natl Cancer Inst. 2005;97(7):536-9.

27. Shen LJ, Wang SY, Xie GF, et al. Subdivision of M category for nasopharyngeal carcinoma with synchronous metastasis: time to expand the $M$ categorization system. Chin J Cancer. 2015;34(10):450-8.

28. Zou X, You R, Liu H, et al. Establishment and validation of M1 stage subdivisions for de novo metastatic nasopharyngeal carcinoma to better predict prognosis and guide treatment. Eur J Cancer. 2017;77:117-26.

29. Sun XS, Liu LT, Liu SL, et al. Identifying optimal candidates for local treatment of the primary tumor among patients with de novo metastatic nasopharyngeal carcinoma: a retrospective cohort study based on Epstein-Barr virus DNA level and tumor response to palliative chemotherapy. BMC Cancer. 2019. https://doi.org/10.1186/s12885-019-5281-5.

30. Li WZ, Lv SH, Liu GY, et al. Development of a prognostic model to identify the suitable definitive radiation therapy candidates in de novo metastatic nasopharyngeal carcinoma: a real-world study. Int J Radiat Oncol Biol Phys. 2021;109(1):120-30.

31. Masterson L, Howard J, Gonzalez-Cruz J, et al. Immune checkpoint inhibitors in advanced nasopharyngeal carcinoma: Beyond an era of chemoradiation? Int J Cancer. 2020;146(8):2305-14.

32. Hsu C, Lee SH, Ejadi S, et al. Safety and antitumor activity of Pembrolizumab in patients with programmed death-ligand 1-positive nasopharyngeal carcinoma: results of the KEYNOTE-028 study. J Clin Oncol. 2017;35(36):4050-6.

33. Ma BBY, Lim WT, Goh BC, et al. Antitumor activity of Nivolumab in recurrent and metastatic nasopharyngeal carcinoma: an international, multicenter study of the Mayo Clinic phase 2 consortium (NCl-9742). J Clin Oncol. 2018;36(14):1412-8.

34. Fang WF, Yang YP, Ma YX, et al. Camrelizumab (SHR-1210) alone or in combination with gemcitabine plus cisplatin for nasopharyngeal carcinoma: results from two single-arm, phase 1 trials. Lancet Oncol. 2018;19(10):1338-50

35. Yang Y, Qu S, Li J, et al. Camrelizumab versus placebo in combination with gemcitabine and cisplatin as first-line treatment for recurrent or metastatic nasopharyngeal carcinoma (CAPTAIN-1st): a multicentre, randomised, double-blind, phase 3 trial. Lancet Oncol. 2021;:S1470-2045(21):00302-8.

\section{Publisher's Note}

Springer Nature remains neutral with regard to jurisdictional claims in published maps and institutional affiliations.

Ready to submit your research? Choose BMC and benefit from:

- fast, convenient online submission

- thorough peer review by experienced researchers in your field

- rapid publication on acceptance

- support for research data, including large and complex data types

- gold Open Access which fosters wider collaboration and increased citations

- maximum visibility for your research: over 100M website views per year

At BMC, research is always in progress.

Learn more biomedcentral.com/submissions 